\title{
A Case of Spontaneous Rupture of Hepatocellular Carcinoma Supplied by the Right Renal Capsular Artery Treated by Transcatheter Arterial Embolization
}

\author{
Joo Yeon Jang', Ung Bae Jeon', Jin Hyeok Kim', Tae Un Kim¹, Hwaseong Ryu', Mong Cho², Young Mi Hong ${ }^{2}$, Maeran Kim \\ Departments of ${ }^{1}$ Radiology and ${ }^{2}$ Internal Medicine, Pusan National University Yangsan Hospital, Pusan National University College of \\ Medicine, Busan, Korea
}

Received Sep. 18, 2018

Revised Nov. 21, 2018

Accepted Dec. 17, 2018
We present a case of spontaneous rupture of hepatocellular carcinoma with poor liver function managed by transcatheter arterial embolization (TAE). The patient's bilirubin level was $2.1 \mathrm{mg} / \mathrm{dL}$, albumin level was $2.4 \mathrm{~g} / \mathrm{dL}$, and prothrombin time international normalized ratio was 2.1. In addition, the patient had also developed a large number of ascites. The tumor was supplied by the right renal capsular artery, as observed on angiography. With successful TAE, no hepatic failure occurred. We believe TAE can be a safe and effective treatment option, even in patients with poor liver function, if tumors are supplied only by extrahepatic collateral vessels. (J Liver Cancer 2019;19:59-63)

Keywords: Hepatocellular carcinoma; Spontaneous rupture; Renal capsular artery; Extrahepatic collateral; Transcatheter arterial embolization

\section{INTRODUCTION}

Transcatheter arterial embolization (TAE) is considered a useful treatment in patients with spontaneously ruptured hepatocellular carcinoma (HCC). ${ }^{1,2}$ However, we must consider the patient's liver function before we embolize the hepatic artery. We report a case of spontaneously ruptured HCC with poor liver function that was successfully managed by TAE via the right renal capsular artery.

\section{Corresponding author: Ung Bae Jeon}

Department of Radiology, Pusan National University Yangsan Hospital, Pusan National University College of Medicine, 20 Geumo-ro, Mulgeumeup, Yangsan 50612, Korea

Tel. +82-55-360-1818, Fax. +82-55-360-1848

E-mail; junwb73@pnuyh.co.kr

https://orcid.org/0000-0002-7731-162X

\section{CASE REPORT}

\section{Clinical findings}

A 53-year-old man with hepatitis B virus infection and alcoholic liver cirrhosis visited the emergency room (ER) in our hospital. He was diagnosed with HCC 3 years ago and received transarterial chemoembolization (TACE) five times, at another hospital. However, he decided to obtain supportive care because his liver function was class $\mathrm{C}$ according to Child-Pugh's classification. Around that time, he visited our ER for regular paracentesis. On that day, he experienced abdominal distension and dyspnea; bloody ascites were diagnosed by paracentesis. His blood pressure was $80 / 40 \mathrm{mmHg}$. Laboratory values on the same day showed a hemoglobin level of $7.3 \mathrm{~g} / \mathrm{dL}$, platelet count of $89 \times 10^{3} / \mu \mathrm{L}$, serum bilirubin level of $2.1 \mathrm{mg} / \mathrm{dL}$, albumin level of $2.4 \mathrm{~g} / \mathrm{dL}$, prothrombin 

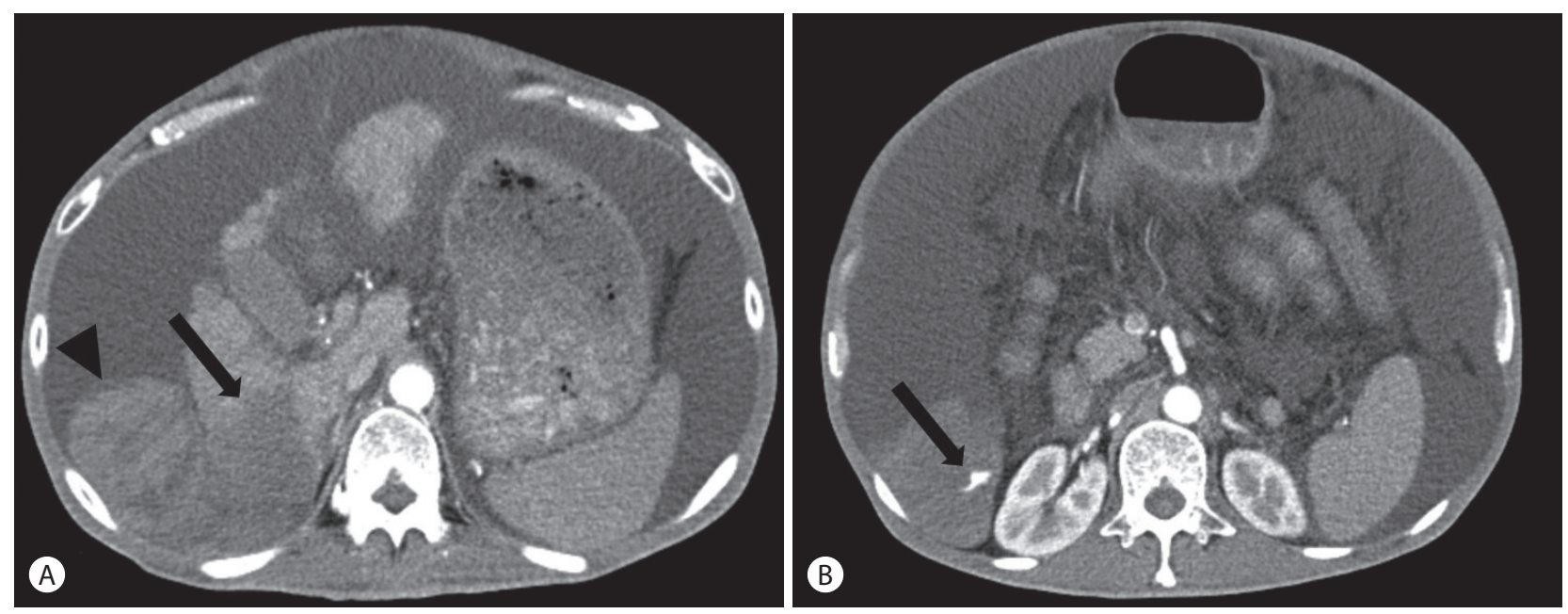

Figure 1. Abdomen computed tomography (CT) findings. (A) CT shows infiltrating hypodense mass (arrow) at the posterior inferior segment (segment 6) of right hepatic lobe with large amount of ascites and hematoma (arrowhead). (B) Arterial phase CT demonstrates extravasation (arrow) of contrast media from this mass and hemoperitoneum.

time international normalized ratio of 2.1, aspartate aminotransferase level of $43 \mathrm{IU} / \mathrm{L}$, and alanine aminotransferase level of $25 \mathrm{IU} / \mathrm{L}$. At that time, there was no evidence of hepatic encephalopathy. Therefore, his Child-Pugh score was class $\mathrm{C}$ at 11 points.

\section{Imaging findings}

Abdomen computed tomography showed an infiltrating hypodense mass at the posterior inferior segment (segment 6) of the right hepatic lobe with tumor thrombosis in the right posterior branch and a large number of ascites (Fig. 1A). In addition, extravasation of contrast media from this mass and hemoperitoneum were observed in the arterial phase (Fig. 1B).

\section{Diagnosis and treatment}

Celiac arteriography using a 5F catheter (Rosch hepatic; Cook Medical Inc., Bloomington, IN, USA) demonstrated a tumor stain in the right lobe of the liver, but without evidence of extravasation (Fig. 2A). Considering that the tumor is located at the periphery of the liver and adjacent to the right kidney, we suspected that the tumor might be supplied by a parasitic blood supply, such as the right renal capsular artery. Right renal arteriography depicted contrast extravasation from the right renal capsular artery (Fig. 2B). Selective arteriography through the right renal capsular artery using a microcatheter (Progreat, Terumo, Tokyo, Japan) and a microwire (Transend, Boston Scientific, Marlborough, MA, USA) revealed a tumor stain and extravasation (Fig. 2C). This artery was embolized with a mixture of $2.0 \mathrm{~mL}$ of iodized oil (Lipiodol Ultrafluid, Guerbet, Aulnay-sous-bois, France) and $0.5 \mathrm{~mL}$ of n-butyl-2-cyanoacrylate (Histoacryl, B. Braun Melsungen AG, Melsungen, Germany) glue. Consequently, the tumor stain and extravasation disappeared (Fig. 2D). After the procedure, the patient's vital signs normalized. His bilirubin value increased to $4.6 \mathrm{mg} / \mathrm{dL}$ transiently, then dropped to below $3.2 \mathrm{mg} / \mathrm{dL}$, and no hepatic failure occurred after TAE. Ten days later, he was discharged. He visited our ER repeatedly because of a large number of ascites and hepatic encephalopathy. He was admitted due to spontaneous bacterial peritonitis. During the hospital stay, the patient developed pneumonia and died about 2 months after undergoing TAE.

\section{DISCUSSION}

Hepatocellular carcinoma is a hypervascular tumor that almost always occurs in patients with liver cirrhosis. It is the 

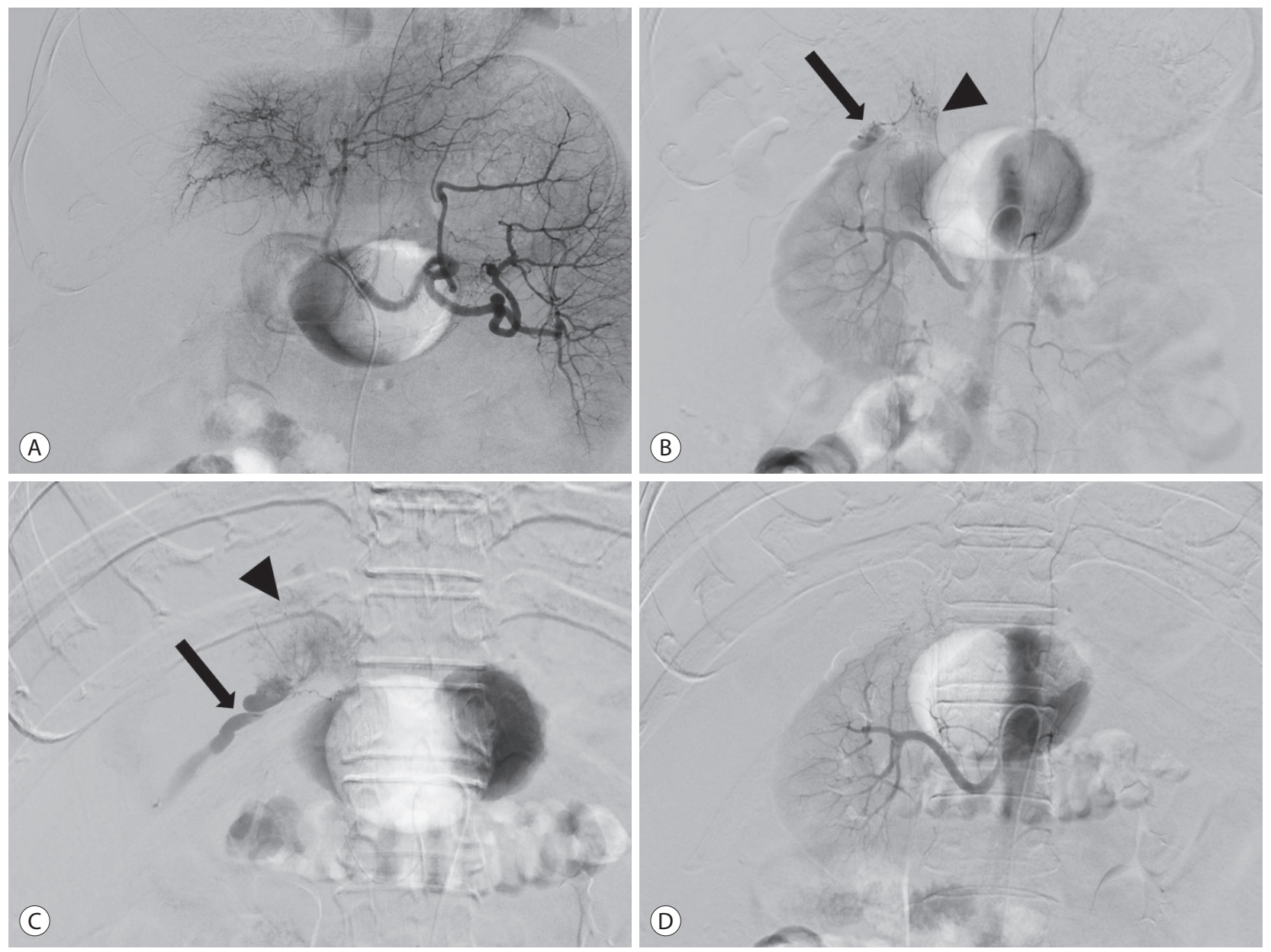

Figure. 2. Transcatheter arterial embolization. (A) Celiac arteriography demonstrates the tumor stain in the right lobe of the liver without evidence of extravasation. (B) Right renal arteriography depicts contrast extravasation (arrow) from right renal capsular artery (arrowhead). (C) Selective arteriography through the right renal capsular artery reveals a tumor stain (arrowhead) and extravasation (arrow). (D) After embolization, the tumor stain and extravasation disappeared.

fifth-most common cancer in the world and its incidence is gradually increasing. ${ }^{3}$ The reported incidence of spontaneous rupture of HCC varies from $2.9 \%$ to $26 \%{ }^{2,4-8}$

The mortality rate in the acute phase of rupture is around $25-75 \%$ of cases. This complication is the third-most common cause of death in HCC patients, after neoplastic progression and liver failure. ${ }^{9}$

The mechanism of spontaneous ruptured HCC is not fully understood. Some investigators believe that disruption of a friable feeding artery or development of a tear in the surface of a tumor subjected to high pressure can cause rupture. Rapid growth of the tumor and necrosis are associated with increased intratumoral pressure caused by progressive or sudden occlusion of branches of hepatic veins due to tumor invasion. ${ }^{4-6,10}$

Several studies have reported that tumor size is significantly greater in spontaneously ruptured HCC than in non-ruptured HCC. ${ }^{7,11}$ When HCC protrudes beyond the original liver margin, the risk of rupture may be higher than that of tumors surrounded by normal liver parenchyma. ${ }^{4,7,12,13}$ Chen et al. ${ }^{7}$ reported that HCC in the left lobe may be more prone to rupture than that in the right lobe.

The symptoms of HCC rupture include the sudden onset of abdominal pain (66.4\%), abdominal distention (16\%), and shock (6.7\%). ${ }^{14}$ Spontaneous rupture is usually a critical and life-threatening condition because of hypovolemic shock 
due to massive blood loss into the peritoneal cavity. The death rate ranges from $1 \%$ to $22 \%$. ${ }^{8,14}$

HCC generally receives blood supply from the hepatic artery, however, various collateral blood supplies have also been reported. ${ }^{15}$ The prevalence of parasitic blood supply is unclear. The right renal capsular artery has also been described as an extrahepatic collateral vessel to the liver. ${ }^{15,16}$

TAE is a useful treatment for unresectable HCC. ${ }^{17,18}$ It causes ischemic necrosis of the tumor tissue in hypervascular HCC. TAE is widely used for various types of HCC unless the main portal vein is occluded. In usual TACE, an iodized oil containing an anticancer drug is used in combination with gelatin sponge particles to act as anti-cancer therapy and to cease bleeding. We use only a gelatin sponge in cases of emergent hepatic artery embolization for HCC rupture in our hospital. In our case, due to bleeding occurring from the parasitic vessel, the sole function of which was to supply the tumor, n-butyl-2-cyanoacrylate was indicated. However, when the parasitic vessel supplies the tumor and the surrounding liver parenchyma, embolic material should be gelatin sponge particles alone.

Acute hepatic failure occurs after TAE in $2.1 \%$ of cases. ${ }^{19}$ The risk factors for acute hepatic failure associated with TAE are poor hepatic functional reserve, a history of multiple embolization procedures, and high doses of chemotherapeutic agents. ${ }^{19}$ Generally, TAE is not performed in patients with poor liver function, especially hyperbilirubinemia $(\geq 2.0$ $\mathrm{mg} / \mathrm{dL}) .{ }^{20}$ Okazaki et al. ${ }^{2}$ argued that emergency TAE for ruptured HCC is contraindicated in patients with hyperbilirubinemia $(\geq 3.0 \mathrm{mg} / \mathrm{dL})$. However, selective TAE reduces the risk of hepatic failure because it minimizes normal liver damage. ${ }^{18}$ It should also be noted that the parasitic blood supply is usually distributed only to the tumor, and not to surrounding hepatic parenchyma. For this reason, TAE via parasitic vessels is safer than that via hepatic arteries in terms of normal liver injury. In our patient, the tumor feeders were both the hepatic artery and right renal capsular artery. However, contrast leakage was observed only in the right renal capsular artery. Therefore, we occluded that artery safely without concerns of acute hepatic failure. In conclusion, we believe that if HCC rupture develops from only extrahepatic collateral vessels, TAE is an effective procedure, even in patients with poor liver function.

\section{ACKNOWLEDGEMENTS}

This study was supported by a 2018 research grant from Pusan National University Yangsan Hospital.

\section{Conflicts of Interest}

The authors have no conflicts to disclose.

\section{REFERENCES}

1. Ngan H, Tso WK, Lai CL, Fan ST. The role of hepatic arterial embolization in the treatment of spontaneous rupture of hepatocellular carcinoma. Clin Radiol 1998;53:338-341.

2. Okazaki M, Higashihara H, Koganemaru F, Nakamura T, Kitsuki H, Hoashi $T$, et al. Intraperitoneal hemorrhage from hepatocellular carcinoma: emergency chemoembolization or embolization. Radiology 1991;180:647-651.

3. Llovet JM, Burroughs A, Bruix J. Hepatocellular carcinoma. Lancet 2003;362:1907-1917.

4. Ong GB, Taw JL. Spontaneous rupture of hepatocellular carcinoma. Br Med J 1972:4:146-149.

5. Nagasue N, Inokuchi K. Spontaneous and traumatic rupture of hepatoma. Br J Surg 1979;66:248-250.

6. Chearanai O, Plengvanit U, Asavanich C, Damrongsak D, Sindhvananda K, Boonyapisit S. Spontaneous rupture of primary hepatoma: report of 63 cases with particular reference to the pathogenesis and rationale treatment by hepatic artery ligation. Cancer 1983; $51: 1532-1536$.

7. Chen CY, Lin XZ, Shin JS, Lin CY, Leow TC, Chen CY, et al. Spontaneous rupture of hepatocellular carcinoma. A review of 141 Taiwanese cases and comparison with nonrupture cases. I Clin Gastroenterol 1995;21:238-242.

8. Liver Cancer Study Group of Japan. Primary liver cancer in Japan. Clinicopathologic features and results of surgical treatment. Ann Surg 1990;211:277-287.

9. Tanaka A, Takeda R, Mukaihara S, Hayakawa K, Shibata T, Itoh K, et al. Treatment of ruptured hepatocellular carcinoma. Int J Clin Oncol 2001;6:291-295.

10. Ong GB, Chu EP, Yu FY, Lee TC. Spontaneous rupture of hepatocellular carcinoma. Br J Surg 1965;52:123-129.

11. Castells L, Moreiras M, Quiroga S, Alvarez-Castells A, Segarra A, Esteban $R$, et al. Hemoperitoneum as a first manifestation of hepatocellular carcinoma in western patients with liver cirrhosis: 
effectiveness of emergency treatment with transcatheter arterial embolization. Dig Dis Sci 2001;46:555-562.

12. Kanematsu M, Imaeda T, Yamawaki Y, Seki M, Goto H, Sone $Y$, et al. Rupture of hepatocellular carcinoma: predictive value of CT findings. AJR Am J Roentgenol 1992;158:1247-1250.

13. Choi BG, Park SH, Byun JY, Jung SE, Choi KH, Han JY. The findings of ruptured hepatocellular carcinoma on helical CT. Br J Radiol 2001;74:142-146.

14. Miyamoto M, Sudo T, Kuyama T. Spontaneous rupture of hepatocellular carcinoma: a review of 172 Japanese cases. Am J Gastroenterol 1991;86:67-71.

15. Charnsangavej C, Chuang VP, Wallace S, Soo CS, Bowers T. Angiographic classification of hepatic arterial collaterals. Radiology 1982;144:485-494.

16. Baba Y, Miyazono N, Inoue H, Kanetsuki I, Nishi H, Nakajo M, et al. Small hepatocellular carcinoma supplied by the right renal cap- sular artery. A case report. Acta Radiol 1999;40:449-450.

17. Yamada R, Sato M, Kawabata M, Nakatsuka H, Nakamura K, Takashima S. Hepatic artery embolization in 120 patients with unresectable hepatoma. Radiology 1983;148:397-401.

18. Uchida H, Ohishi H, Matsuo N, Nishimine K, Ohue S, Nishimura $Y$, et al. Transcatheter hepatic segmental arterial embolization using lipiodol mixed with an anticancer drug and Gelfoam particles for hepatocellular carcinoma. Cardiovasc Intervent Radiol 1990;13:140-145.

19. Katsushima S, Inokuma T, Oi H, Okamura J, Higashi T, Takeuchi R, et al. Acute hepatic failure following transcatheter arterial embolization for the treatment of hepatocellular carcinoma. Digestion 1997;58:189-195.

20. Oi H. Liver intervention. Nihon Igaku Hoshasen Gakkai Zasshi 2000;60:826-832. 